\title{
L'importanza del comparto socio-economico per uno sviluppo sostenibile: il caso studio dell'aeroporto Antonio Canova di Treviso
}

\author{
Francesco Calicchio $^{1}$ and Catello Di Martino ${ }^{1}$
}

${ }^{1}$ Affiliation not available

\begin{abstract}
Nel corso del tempo e soprattutto dall'introduzione del concetto di sostenibilità, il comparto socio-economico e le leggi che lo governano rientrano in pieno nella tematica, divenendo elemento essenziale ed imprescindibile.

Per l'assetto economico, la relazione con le altre componenti è determinata dall'interferenza reciproca tra le caratteristiche delle componenti ambientali (stato di qualità dell'aria, qualità delle acque, caratteristiche del suolo, ecc.... ) i fattori ambientali individuati (rumore, vibrazione, traffico, radiazioni, rifiuti, energia, ecc.) e le caratteristiche del sistema produttivo. Le caratteristiche delle altre componenti e degli altri fattori ambientali possono infatti condizionare la sostenibilità ambientale del sistema produttivo, così come il sistema produttivo può condizionare la sostenibilità ambientale (ad esempio, presenza di attività industriali che comportano rilevanti emissioni inquinanti in atmosfera condizionerà lo stato di qualità dell'aria).
\end{abstract}

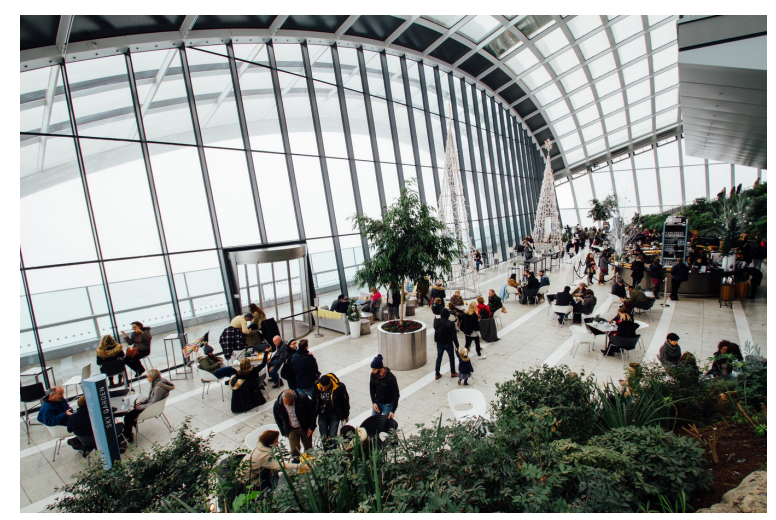

Figure 1: Flusso turistico all'interno dell' Aeroporto di Londra, Gran Bretagna. 


\section{Comparto socio-economico: la sua valenza all'interno della tematica della sostenibilità}

In tempi moderni, le componenti socio-economiche sono entrate giocoforza a far parte intrinsecamente del concetto di sostenibilità; le stesse, infatti, vengono intese come quegli elementi che hanno la capacità di creare una crescita duratura degli indicatori economici, generare reddito e lavoro, sostenere le popolazioni, valorizzarne le specificità territoriali e allocarne efficacemente le risorse.

Nonostante il comparto socio-economico abbia una forte rilevanza, non è disciplinato dal D.P.C.M. 27/12/1988. Di conseguenza, è necessario inquadrare il comparto all'interno del concetto di sviluppo sostenibile.

Quest'ultimo, nel tempo, è stato oggetto di critiche: tra le tante vi è quella secondo cui è impossibile pensare che uno sviluppo economico sia basato sui continui incrementi di produzione e sia compatibile allo stesso tempo con la preservazione dell'ambiente.

Per garantire equilibrio e coesistenza fra le componenti alla base della società sono stati introdotti degli indicatori a carattere globale. Quest'ultimi sono delle grandezze economiche che forniscono informazioni sull'attività economica di un Paese. Gli indicatori economici analizzati vengono riassunti e descritti all'interno delle seguenti tabelle:

\begin{tabular}{|c|c|}
\hline Indicatori & Descrizione \\
\hline 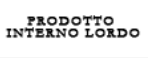 & $\begin{array}{l}\text { Indicatore delle prestazioni di un'economia } \\
\text { nel corso del tempo. }\end{array}$ \\
\hline PRODDATO & Rapporto tra il PIL e il numero di cittadini. \\
\hline 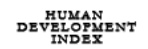 & $\begin{array}{l}\text { Unità di misura per il confronto delle condizioni } \\
\text { socio-economiche dei vari Paesi del mondo. }\end{array}$ \\
\hline
\end{tabular}

Figure 2: Principali indicatori economici generalmente a livello locale o nazionale.

*Tutte le tabelle sono oggetto di rielaborazione personale. 


\begin{tabular}{|l|l|}
\hline $\begin{array}{c}\text { Indicatori } \\
\text { INDICE DI SVILUPPO } \\
\text { PER GENERE }\end{array}$ & Non fornisce indicazione sulle discriminazioni. \\
$\begin{array}{l}\text { INDICE DR } \\
\text { POVERTA UMANA }\end{array}$ & $\begin{array}{l}\text { Valuta se gli individui dispongano o meno delle } \\
\text { opportunita neccesarie per godere di un tenore } \\
\text { di vita accettabile. }\end{array}$ \\
\hline
\end{tabular}

Figure 3: Altri indicatori economici a carattere globale.

\begin{tabular}{|c|c|}
\hline Principali Indicatori per $1 \mathrm{a}$ VIA e $1 \mathrm{a}$ VAS \\
POPOLAZIONE & $\begin{array}{c}\text { COMUNITA' } \\
\text { ED } \\
\text { ISTITUZIONI }\end{array}$ \\
$\begin{array}{c}\text { INDIVIDUI } \\
\text { E } \\
\text { FAMIGLIE }\end{array}$ & ECONOMIA \\
\hline
\end{tabular}

Figure 4: Principali indicatori economici per la VIA e la VAS.

\section{Caso studio: l'Aeroporto “Antonio Canova di Treviso"}

L'aeroporto Antonio Canova si trova a $3 \mathrm{~km}$ dal centro della città di Treviso, in una zona adiacente alla tangenziale della città che lo collega in pochi minuti alle principali reti autostradali. La sua pista ha una lunghezza di $2.459 \mathrm{~m}$ il che lo colloca al terzo posto in Italia dopo gli aeroporti di Roma e Milano.

Nel 2011 il Masterplan dell'Aeroporto Antonio Canova di Treviso è stato soggetto alla procedura di Valutazione di Impatto Ambientale - VIA - in particolare in quanto riconducibile alle categorie di cui all'allegato II "Progetti di competenza statale, ovvero aeroporti con piste di atterraggio superiori a 1.500 metri di lunghezza".

II Masterplan viene inoltre sottoposto congiuntamente, nell'ambito della stessa procedura di VIA, a Valutazione di incidenza, per cui viene prevista l'elaborazione di un documento distinto (Relazione di Valutazione di incidenza), ma coerente nei contenuti al SIA, in cui vengono trattati specificamente gli effetti del Masterplan su habitat e specie presenti nei siti della Rete Natura 2000 limitrofi all'aeroporto.

Nel presente caso studio viene inquadrata la componente socio-economica, relativamente agli aspetti direttamente ed indirettamente interferiti dalle azioni e dagli elementi costituenti lo Strumento di pianificazione e ottimizzazione al 2030 dell'aeroporto "A. Canova" di Treviso. Scopo della presente analisi è quello di valutare, attraverso uno studio qualitativo previsionale, gli effetti di carattere economico e sociale che verranno a manifestarsi, nel contesto locale, a seguito di un ipotizzato aumento del traffico aereo an- 
nuo, in termini di decolli/atterraggi e conseguente variazione quantitativa di passeggeri, previsto per l'aeroporto. Lo scalo aeroportuale ha conosciuto nell'ultimo decennio un'importante crescita. Tale espansione ha generato un notevole indotto economico per l'intero contesto territoriale in cui lo scalo si situa, soprattutto in termini occupazionali e in termini legati al turismo e aspetti logistici.

Nella definizione dell' area di studio per l'analisi socio economica si è scelto di limitarsi all'area provinciale, base territoriale per la quale sono disponibili dati utili alla caratterizzazione della componente. L'area vasta verrà poi confermata nelle successive valutazioni e si limita ai territori dei comuni in cui si sviluppa il sedime aeroportuale e cioè Treviso, Quinto di Treviso e Zero Branco.

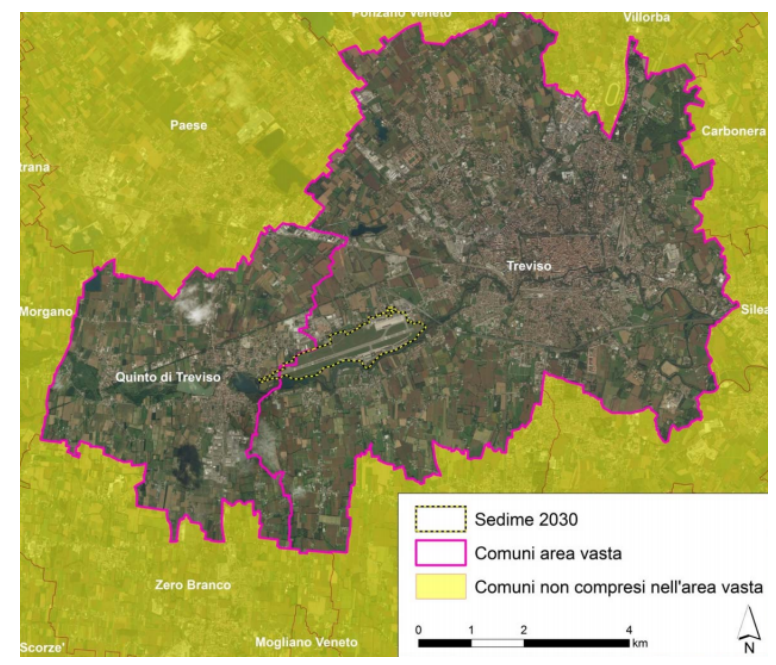

Figure 5: Comuni compresi nell'area vasta per la componente socioeconomica. Link

In seguito, sono stati analizzati alcuni dati statistici riguardanti i principali aspetti demografici e sociali dell'area studio. Gli indicatori analizzati sono stati:

\section{-Età della popolazione:}

Tra le principali trasformazioni demografiche degli ultimi anni è da considerare l'invecchiamento della popolazione;

\section{- Occupazione:}

Caratterizzata dalla disoccupazione e dalla contrazione nel numero delle imprese;

\section{- Stranieri nel territorio:}


Gli stranieri rappresentano una percentuale che oscilla tra l'11 ed il $12 \%$ della popolazione totale;

\section{- Andamento della popolazione:}

La popolazione complessiva dei comuni risulta in crescita rispetto i primi anni del 2000 .

II saldo naturale negativo, ovvero con la natalità che è inferiore alle mortalità, viene compensato dal fenomeno migratorio che determina una popolazione tendenzialmente in lieve crescita.

Per quanto riguarda gli impatti socio-economici, correlati all'attività aeroportuale, uno dei possibili metodi di approccio è quello basato sull'analisi costo - benefici.

Nel seguito, viene presentata una breve analisi qualitativa che evidenzia quali siano i maggiori benefici e le criticità legate allo sviluppo aeroportuale.

Quindi, potremmo avere diverse tipologie di impatto:

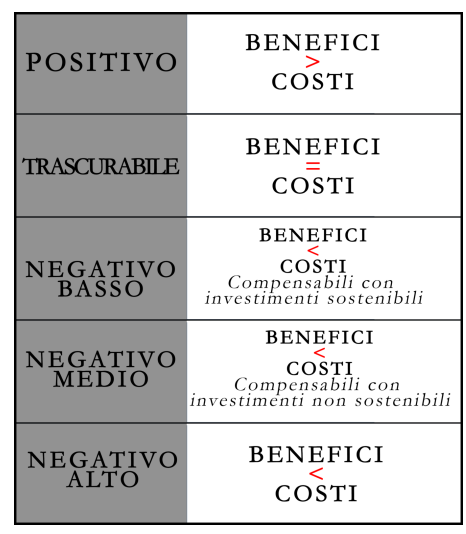

Figure 6: Le diverse tipologie di impatto.

Benefici economici stimabili e correlabili allo scalo aeroportuale, sono quelli legati all'aspetto occupazionale. Lo sviluppo dell'attività aeroportuale infatti, oltre ad avere effetti significativi di tipo occupazionale per i settori legati alle funzioni aeroportuali, stimola anche quelle attività economicoproduttive produttrici di beni e servizi non localizzati nelle immediate vicinanze. Secondo la distinzione proposta da uno studio eseguito dall' $\mathrm{ACl}$ Europe 2004, i benefici in termini occupazionali si possono distinguere in:

Sempre dagli studi effettuati dall'ACl Europe, in previsione dello scenario 2030 si può raggiungere la cifra di oltre 1.500 posti di lavoro.

Quindi, la somma dell'occupazione diretta, dell'occupazione indiretta e dell'occupazione indotta determina l'incremento del PIL dell'area di riferi- 


\begin{tabular}{|c|c|c|}
\hline OCCUPAZIONE & $\begin{array}{c}\text { OCCUPAZIONE } \\
\text { DIRBTTA }\end{array}$ & $\begin{array}{c}\text { OCCUPAZIONE } \\
\text { INDOTTA }\end{array}$ \\
$\begin{array}{c}\text { Generata dalla presenza } \\
\text { degli scali nel } \\
\text { territorio. }\end{array}$ & $\begin{array}{c}\text { Nasce dallo sviluppo di } \\
\text { attività economiche di } \\
\text { fornitura al settore del } \\
\text { trasporto aereo. }\end{array}$ & $\begin{array}{c}\text { Supporta l'attività di negozi, } \\
\text { imprese produttrici di beni di } \\
\text { consumo ed imprese di altri } \\
\text { settori dei servizi. }\end{array}$ \\
\hline
\end{tabular}

Figure 7: Le diverse tipologie di occupazione.

mento per una quota percentuale che varia tra l' $1.4 \%$ e il $2.5 \%$ (escludendo gli impatti occupazionali derivanti dal turismo).

Nel corso dell' analisi costi-benefici si è arrivati a dedurre che le componenti di costo analizzate sono due: costi di carattere economico e costi di carattere ambientale.

Nei costi di carattere economico rientrano tutte le spese per il potenziamento ed ammodernamento dell'aeroporto e i relativi costi degli interventi intrapresi, compresi quelli al fine di prevenire o ridurre eventuali danni all'ambiente derivanti dalle attività operative. I costi di carattere economico, a carico del gestore dell'aeroporto, hanno un importo a pari a circa 53 milioni di euro, comprensivi dei costi per le opere di mitigazione e compensazione, che incidono del $7 \%$ sui costi economici totali.

I costi di carattere ambientale, invece, non sono direttamente correlati ad un valore economico e possono essere legati, ad esempio, all'incremento del traffico, all'aumento del traffico dei veicoli nelle vicinanze dell'aeroporto con conseguente congestione stradale e alle manovre degli aeromobili e dei mezzi di servizio.

La componente viene condizionata positivamente dalle opere di compensazione e mitigazione per quanto riguarda i comparti di atmosfera e rumore. Per le misure di mitigazione si è proceduto con l'isolamento degli edifici. Per quanto riguarda, invece, le misure di compensazione si è proceduto con interventi di riparazione delle coperture danneggiate, ma soprattutto di consolidamento preventivo di quelle a maggior rischio di fenomeni "vortex strike" e allo spostamento della scuola materna S. Giorgio. Tutti gli interventi hanno un costo pari a 3.900 .000 euro.

*Tutte le tabelle sono oggetto di rielaborazione personale.

\section{Conclusioni}

In tutti gli Scenari analizzati, i costi ambientali legati all'attività aeroportuale che possono influenzare in maniera negativa la componente socioeconomica sono sostanzialmente riconducibili agli aspetti legati al rumore. Tale problematica rappresenta evidentemente uno dei problemi chiave connessi alla presenza di una struttura aeroportuale per quanto riguarda le emissioni sonore da decollo e atterraggio aereo. Tali impatti, strettamente correlati 
alla componente salute pubblica, possono interagire con la componente socioeconomica determinando una diminuzione del benessere e della qualità della vita dei cittadini residenti nelle zone limitrofe l'aeroporto.

L'incremento della mobilità aerea può contribuire ad aumentare il livello di efficienza e di produttività delle aziende creando $i$ presupposti per un facile accesso alle aziende fornitrici e ai consumatori finali.

Si è arrivati alla conclusione che lo scenario 2030 si ritiene positivo per il comparto socio-economico rispetto sia allo stato di fatto che scenario di riferimento. In assenza degli investimenti previsti sulle opere di mitigazione e compensazione, lo stato di fatto rispetto allo scenario di riferimento si ritiene trascurabile.

\section{References:}

1. "Strumento di pianificazione e ottimizzazione al 2030 dell'aeroporto "A. Canova" di Treviso Studio di Impatto Ambientale Sezione C Quadro di riferimento ambientale SOCIO-ECONOMIA" Link

2. "Strumento di pianificazione e ottimizzazione al 2030 dell'aeroporto "A. Canova" di Treviso Procedura di Valutazione di Impatto Ambientale INQUADRAMENTO GENERALE ALLA DOCUMENTAZIONE PRESENTATA" Link

3. "Strumento di pianificazione e ottimizzazione al 2030 dell'aeroporto "A. Canova" di Treviso Studio di Impatto Ambientale Sezione C Quadro di riferimento ambientale MITIGAZIONI E COMPENSAZIONI, MONITORAGGIO E CONCLUSIONI"' Link

4. "ACl Europe, 2004. The social and economic impact of airports in Europe." Link

5. "Provincia di Treviso, 2013. Sintesi delle tendenze demografiche, economiche e sociali in Provincia di Treviso. Relazione a cura dell'Ufficio Statistico della Provincia di Treviso, Settore Protezione Civile, Caccia, Pesca e Agricoltura". Link

6. "Camera di Commercio di Treviso 2015b. La demografia di impresa a Treviso nel 2014". Link

7. "Aeroporto di Treviso "Antonio Canova" MASTERPLAN Relazione e Piano degli investimenti, 2011". Link

8. Aeroporto di Londra - Foto di Riccardo Bresciani da Pexels; Link

9. V.Naddeo, V.Belgiorno. Tecniche per la valutazione di impatto ambientale. ASTER onlus Editore, 2011. 